\title{
REFLEXIONES ACERCA DEL PROCESO PSICO-SOCIAL DE VALORACION DE IDONEIDAD PARA LA ADOPCION DE MENORES EN LA COMUNIDAD VALENCIANA
}

\author{
ANA ROSSER LIMIÑANA \\ Departamento de Comunicación y Psicología Social. Universidad de Alicante.
}

\section{RESUMEN}

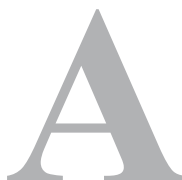

lo largo de este trabajo, se realiza un análisis de los criterios técnicos y legales que sustentan la necesidad de realizar un proceso de valoración psico-social de las familias solicitantes de adopción, de los elementos que lo configuran y que pasan por distintas fases, desde que la familia se plantea la

adopción hasta que el menor se incorpora definitivamente a su nueva familia, así como de sus resultados y consecuencias, haciendo una serie de consideraciones sobre las implicaciones de una resolución de no idoneidad. Finalmente, y a modo de conclusión, se propugna un modelo de trabajo conjunto con las familias, que permita la reflexión sobre sus expectativas y sus capacidades para la paternidad adoptiva, y la necesidad de seguir unos criterios que, alejados de posturas demasiado rígidas, sean capaces de adaptarse a la realidad social cambiante y a contextualizar las demandas de las familias y de adecuarlas a las necesidades actuales de los menores.

Palabras clave: adopción, paternidad adoptiva, valoración psicosocial.

\section{ABSTRACT}

This paper analyses the technical and legal criteria that justify the need for a psychosocial evaluation process for families requesting adoption, the elements that it would contain and the stages involved, from families considering adoption through to when the child is definitively incorporated into its new family, and the results and consequences thereof, making a series of considerations concerning the implications of a ruling of non-eligibility. Finally, and by way of conclusion, a model is proposed for working with families in a way that considers both their expectations and their capacity for adoptive parenthood, and the need to follow criteria that are flexible enough to adapt to changing social circumstances, and to put family demands into context and adapt them to the children's current needs.

Key words: adoption, adoptive parenthood, psychosocial evaluation. 


\section{JUSTIFICACIÓN: CRITERIOS TÉCNICOS Y MARCO LEGAL}

El tema de este trabajo ha sido ya abordado ampliamente y se puede plantear desde distintos puntos de vista, según pongamos el enfoque en unos u otros protagonistas del proceso adoptivo. Nuestra aportación se apoya en la idea de que, elijamos el punto de vista que elijamos, todos justificarían que se efectúe un proceso de valoración de la idoneidad de las familias que desean adoptar.

En una de estas posiciones, se encuentran los menores: aquellos niños y niñas sobre los que, como consecuencia de una serie de circunstancias familiares y sociales, los técnicos de los Servicios de Protección de Menores deciden su adopción, con la finalidad de ofrecerles una familia que les acoja, les cuide, y vele por ellos. En la otra, están las familias que acuden a los Servicios de Adopción porque desean tener un hijo y se encuentran con una serie de obstáculos no previstos. Para acceder a su deseo, las entidades competentes les piden que se sometan a un proceso de valoración, que muestren cómo son, cómo viven, sus deseos y necesidades, y que cuenten con que, al adoptar, deben asumir las necesidades de un niño que va a llegar a su hogar junto con una serie de experiencias, de privaciones, de carencias, a las que deben ser capaces de dar respuesta. Por último, se encuentran las entidades públicas, las de nuestro país y las del suyo (si se trata de una adopción trans-nacional), cuya responsabilidad es la de poner todos los medios para que los distintos planteamientos, las distintas necesidades encajen, para que realmente las familias seleccionadas para cubrir las necesidades de esos menores puedan cumplir su cometido de forma exitosa y para que esos niños hagan que las familias se sientan realizadas en su papel de padres y madres. En esa tarea, las entidades públicas cuentan frecuentemente con la colaboración de las Entidades Colaboradoras de Adopción Internacional (en adelante, E.C.A.I.s.), que contactan con las familias, las orientan y las acompañan en todo el proceso, cuando la adopción se realiza en otro país.

Desde el prisma de los menores, observamos una serie de elementos diferenciales entre sus vivencias y las de cualquier otro niño que van a hacer necesario que sus futuros padres cuenten con determinados conocimientos, capacidades y habilidades. Todos han tenido y tienen una familia de origen, unas raíces y un pasado que estarán de alguna forma presentes a lo largo de su vida, una familia con la que, probablemente, existieron unos lazos efectivos y sobre la que el menor en algún momento querrá saber cómo y por qué fue que lo abandonaron, qué fue de ellos,... Con gran probabilidad, antes de llegar a los sistemas de protección, habrán pasado privaciones, carencias, quizá experiencias de maltrato o negligencia que han dejado su huella y pueden manifestarse en trastornos de salud, problemas de desarrollo, alteraciones emocionales y/o del comportamiento, retraso escolar, etc. Si se han visto sometidos a separaciones efectivas de sus personas de referencia, pueden sufrir, como consecuencia de estas, reacciones emocionales que dificulten su vinculación afectiva a unas nuevas figuras. Si los menores han sido adoptados a través de la adopción internacional, nos encontramos, además, en la mayoría de los casos, con niños y niñas pertenecientes a etnias y culturas diferentes, con rasgos distintos, distintas costumbres y un idioma, en ocasiones, también distinto que puede suponer un obstáculo añadido en su adaptación. En definitiva, son niños como los demás, que necesitan los mismos cuidados $\mathrm{y}$ atenciones que cualquier otro, pero que, por distintas razones, no los tuvieron. Sus experiencias previas, sus sentimientos y sus vivencias impregnarán inevitablemente el proceso de integración en su nueva familia.

Ello va a comportar, por tanto, importantes diferencias en el desempeño de la paternidad por parte de quienes van a ser sus nuevos padres. Diferencias que se ven acentuadas al tener que hacer frente a circunstancias que no son habituales en otras familias, lo que puede suponer una situación de estrés y vulnerabilidad específica (Generalitat Valenciana, 1999b), que, en ocasiones, si no es superada, conduce al fracaso del proyecto adoptivo. 
Esta realidad, la de los menores y la de las familias, es la que justifica la necesidad de que entren en juego terceras personas -las entidades eúblicas y las agencias colaboradoras (ECAIS) - para llevar a cabo un proceso de valoración y selección de las familias que quieren tener un hijo a través de la adopción.

La responsabilidad de las entidades implicadas es preparar a las familias y hacer una valoración de su realidad psicológica y social tan completa que permita posteriormente seleccionar a una familia que realmente pueda cubrir las necesidades de ese niño, garantizando así al menor que la familia que se le ofrece va a ser capaz de afrontar su crianza.

Esta necesidad se ha visto corroborada por años de experiencia en los que se ha puesto de relieve la existencia de importantes crisis familiares en familias adoptivas (Groze, V., 1994; Palacios, J., Sánchez, Y. y León, E., 2002 y 2005; Berástegui, A., 2003). Pero, también, porque contamos con estudios (Rosemboom, 1991; Triseliotis, J., Shireman, J., Hundleby, M., 1997; Berástegui, A., 2003; Mestre, M.V., Samper, P. y Tur, A.M., 2008,) que destacan las características tanto de los menores como de las familias que permiten pronosticar una mayor probabilidad de éxito en el proceso adoptivo y que, en el caso de estas últimas, van desde aspectos como la motivación y expectativas de los solicitantes, sus recursos educativos, sus actitudes, el ajuste de pareja, el nivel de estrés, etc., válidos para todos, hasta otras cuestiones más concretas referidas a la edad, las aficiones, la existencia de otros hijos, etcétera, que nos permiten una mejor elección si las tenemos en cuenta.

Si su importancia es incuestionable en el ámbito de la adopción nacional donde los profesionales conocen a los menores y conocen a las familias candidatas, tanto más en la adopción internacional, donde tenemos el deber de garantizar a los profesionales encargados de las asignaciones, en los países de origen, todos los elementos para que estas sean certeras, a pesar de la distancia tanto física corno temporal de las familias candidatas.

En los últimos años, además, gracias a las aportaciones de la Teoría del Apego y de la Resiliencia al ámbito de la adopción (Bowby, 1985; Marrone, M., 2001; Legaz, E, 2003; Cyrulnick, B., 2002), se ha hecho especial hincapié en los procesos de valoración en la historia vincular de los futuros padres y su capacidad para, a través de factores resilientes, compensar los déficits que pueden presentar los menores adoptados, ayudándoles a reparar sus heridas y a generar recursos para desarrollarse óptimamente y vincularse afectivamente a su nueva familia.

Todos estos planteamientos cuentan con un marco legal que sirve de soporte al procedimiento técnico y que adjudican a la entidad pública la responsabilidad del proceso. El punto de partida es la Convención de los Derechos del Niño (1989) que, en su preámbulo, reconoce que el niño, para el pleno y armonioso desarrollo de su personalidad, debe crecer en el seno de una familia, y reconoce la adopción como forma alternativa de vida familiar y la adopción internacional como recurso subsidiario, cuando no es posible su colocación en una familia de su propio país. Posteriormente, el Convenio de la Haya, de 29 de mayo de 1993, relativo a la protección del niño y a la cooperación en materia de adopción internacional, que fue ratificado por España en 1995, toma en consideración los principios allí recogidos y nace con el objetivo, entre otros, de establecer las garantías necesarias para que la adopción internacional tenga lugar en consideración al interés del niño y al respeto de los derechos fundamentales que le reconoce el derecho internacional. Con esta finalidad, el convenio señala, en su artículo 17, que el Estado de origen solo podrá confiar al niño a los futuros padres adoptivos si se ha constatado que son adecuados y aptos para adoptar. Igualmente, señala cuáles serían las competencias tanto de las entidades públicas como de las entidades colaboradoras en las distintas fases del proceso. A su vez, todos estos principios son recogidos en la nominativa estatal con la L.O. 1/1996 de Protección Jurídica del Menor que, en su exposición de motivos, hace especial hincapié en la obligatoriedad de la entidad pública de ofrecer al menor una alternativa familiar, cuando no puede contar con la suya propia, y modifica el artículo 9.5 del Código Civil, estableciendo la necesidad de 
la idoneidad de los adoptantes para acceder a la adopción y que sea reconocida en España una adopción constituida en el extranjero. Y, recientemente, la Ley 54/2007 de adopción internacional precisará más estos preceptos, especialmente en lo referido a la intervención de las entidades públicas y la intermediación de las entidades colaboradoras en el proceso de adopción y, en concreto, el requisito de la idoneidad para adoptar.

En nuestra Comunidad, la Ley 12/2008, de 3 de julio de 2008, de la Generalitat, de Protección Integral de la Infancia y la Adolescencia de la Comunitat Valenciana determinará las competencias de la Generalitat en los procedimientos de adopción internacional, establecerá el procedimiento para la valoración de la idoneidad de los solicitantes de adopción internacional y fijará los criterios para otorgar la conformidad a las pre-asignaciones.

Sin duda, el consenso en la necesidad de realizar un proceso de valoración psico-social, previo a la declaración de la idoneidad para la adopción y la selección de las familias candidatas, es unánime. Pero es importante analizar cómo y de qué manera se pueden lograr estos objetivos.

\section{ELEMENTOS DEL PROCESO PSICO-SOCIAL DE VALORACIÓN DE LAS FAMILIAS ADOPTANTES}

El reconocimiento tanto legal como técnico de la necesidad de valorar la idoneidad de las familias para la adopción exige que, desde la Administración, exista un procedimiento para valorar a las familias y así poder informar a las personas encargadas de la selección de las familias sobre el perfil de los solicitantes de adopción, elaborando, como señala el artículo 16 del Convenio de la Haya, un informe que contenga información sobre su identidad, capacidad jurídica y aptitud para adoptar, su situación personal, familiar y médica, su medio social, los motivos que le animan, su aptitud para asumir una adopción y sobre los niños que estarían en condiciones de tener a su cargo.

Sin embargo, también ha llevado a que una de las preocupaciones de los futuros candidatos a ser padres y madres adoptivos surja del temor, más o menos reconocido, que muchas personas sienten al tener que afrontar las diversas entrevistas y evaluaciones psico-sociales que les realizan los equipos de adopción. El someterse a un «examen» por unos desconocidos es algo poco agradable para la mayoría, especialmente si de la «nota final» depende el ver o no ver finalmente cumplidas las expectativas como padres.

Aunque es una idea difícil de trasmitir a estas familias, desde la entidad pública, el certificado de idoneidad se concibe como el último peldaño de un proceso de preparación y valoración (Amorós, P., 1987; Amorós P., Fuertes, 1996) que empezó cuando se plantearon la adopción y que se fundamenta en la necesidad de informar y orientar a las familias, para que puedan conocer y reflexionar sobre las peculiaridades de la paternidad adoptiva, valorando conjuntamente con ellos su motivación, necesidades y actitudes y preparándoles para afrontar las situaciones con las que se pueden encontrar a lo largo del proceso de adopción de su hijo (Generalitat Valenciana, 1999a).

Con este objetivo, la valoración de las familias adoptivas se desarrolla en distintas etapas, en las que se trata de clarificar sus ideas, romper sus prejuicios, valorar aspectos positivos y negativos de su proyecto y, en definitiva, calibrar sus posibilidades de éxito.

El proceso se inicia con una primera fase puramente informativa en la que las familias recopilan todos los datos necesarios sobre procedimiento, plazos, alternativas, costes, entidades de mediación (en el caso de las adopciones internacionales), etcétera y que suele concluir con la solicitud de adopción y la apertura de su expediente.

Le sigue, a continuación, la fase de preparación-valoración, que entendemos es una sola, aunque está conformada por dos etapas distintas. La primera de ellas, caracterizada 


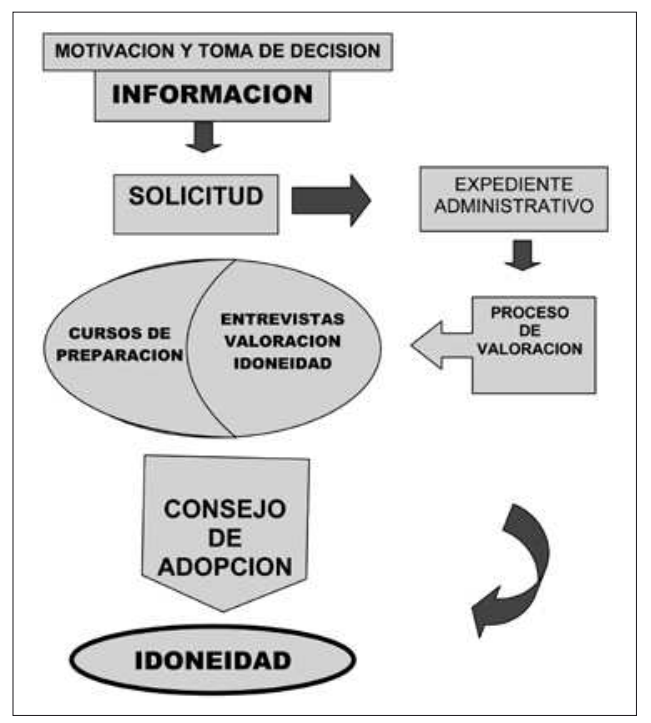

FIGURA 1: Proceso de valoración psico-social de la idoneidad para adoptar.

por la participación en unos talleres de formación, y la segunda, con las entrevistas de valoración psico-social.

La preparación-formación se realizará a través de actividades grupales, a lo largo de varias sesiones, en las que se abordan cuestiones relacionadas con la motivación y expectativas de los futuros padres, las características de los menores y de sus familias de origen, la necesidad y la manera de abordar la revelación de la condición de adoptado, situaciones propias de la convivencia en los procesos adoptivos y sobre las habilidades necesarias para abordarlas, etc.

Siguiendo a Fuertes y Amorós (1.997), los grupos de formación suelen atender a tres aspectos de los participantes:

1. Aspectos actitudinales y emocionales, como es la disposición a aceptar el pasado del niño, sus sentimientos y recuerdos sobre su familia, la disposición a mostrar respeto hacia la familia biológica y las circunstancias que llevaron a la separación definitiva, a ayudar al niño a conservar y valorar su propia historia, a aceptar los sentimientos del niño de ambivalencia, inseguridad y sus deseos de conocer más acerca de su genealogía, etcétera.

2. Desarrollo de habilidades que permitan afrontar de forma competente la tarea de adecuar a un niño adoptado o a un niño con problemas de comportamiento, en su caso.

3. Aspectos cognitivos relacionados con el proceso de la adopción y sus implicaciones, los problemas más habituales, los recursos existentes en la comunidad, etc.

En este sentido, estudios realizados sobre el impacto de estos talleres de adopción (Moya, C., Rosser, González, I., 1999; Rosser, A., Moya, C., González, L., 2000) han corroborado que las actividades de formación con las familias generaban no sólo un mayor conocimiento de las particularidades del proceso adoptivo sino también un cambio en las actitudes de los candidatos respecto a varias cuestiones importantes para el éxito en la adopción.

Concluida la fase de preparación, se inicia la siguiente etapa, ya propia de la valoración psico-social que permitirá, no sólo conocer las características de las personas que desean 
adoptar sino también, profundizar sobre los temas tratados en los talleres, resolver dudas, ajustar expectativas, etc. Para realizar esta exploración, los profesionales del ámbito de la Psicología y el Trabajo Social, a través de entrevistas individuales y conjuntas, visitas domiciliarias, cuestionarios de personalidad, etcétera, irán ahondando en los diferentes aspectos considerados relevantes para el éxito del proceso adoptivo. La valoración psicosocial de idoneidad para la adopción concluye con unos informes psicológicos y sociales que recogen datos referidos a la historia personal y de la pareja, en su caso, su motivación y expectativas respecto a la adopción, su entorno socio-familiar, su situación económica y laboral, sus capacidades educativas, sus perfiles de personalidad, su estado de salud, etc. (Generalitat Valenciana, 1999a) y una valoración sobre su adecuación para hacer frente a una adopción, remarcando aquellos aspectos del contenido del informe que sustentan esa decisión y apuntando, en los casos favorables, hacia el perfil del niño para el que se considera, en su caso, más adecuada la familia, al objeto de poder orientar una futura asignación.

El proceso de valoración psico-social, tal y como está establecido en la Comunidad Valenciana, finalizará con el acuerdo que emite el Consejo de Adopción de Menores de otorgar, en su caso, el Certificado de Idoneidad a la familia para la adopción.

Desde ese momento, y una vez se trasladan los informes a los países de origen en el caso de la adopción internacional, las familias quedan a la espera de ser seleccionadas para un caso en particular.

En concreto, en las adopciones internacionales, en base a la información obrante en estos informes y remitida a las entidades competentes, proseguirá el proceso hasta la adopción. Se procederá, por parte de los países de origen, a la asignación de un menor a la familia. En nuestro país, se recabará la aceptación, en su caso, tanto de la familia como de la entidad pública y, finalmente, con el viaje de los futuros padres, culminarán los trámites judiciales para la adopción y el menor se incorporará definitivamente a su nuevo hogar.

Si la adopción se realiza en nuestro país, el proceso será muy similar, aunque todo se efectuará en la propia entidad pública.

Finalmente, un vez incorporado el menor a su familia, los profesionales de los servicios de adopción realizarán el seguimiento de los procesos de adaptación entre los menores y su familia, informando, en su caso, a los países de origen sobre la evolución de los mismos y apoyando a las familias en las dificultades con las que pudieran encontrarse.

\section{RESULTADO DEL PROCESO DE VALORACION PSICO-SOCIAL Y SUS CONSECUENCIAS}

En general, el resultado del proceso de valoración psico-social es la obtención del certificado de idoneidad para la adopción, que suele ir acompañado, en el cuerpo del informe, por las características del menor para el que se valora apta a la familia solicitante y que permite concluir el expediente recabando la documentación requerida por los distintos países.

Por tanto, como señalábamos anteriormente, el informe psico-social de idoneidad se convierte en un elemento crucial para facilitar la asignación y el "matching" adecuado entre menores y familias.

Sin embargo, no siempre ocurre así. En ocasiones, la valoración realizada por los profesionales es negativa y el Consejo de Adopción de Menores emite una resolución de no idoneidad.

Las implicaciones de esta Resolución requieren que nos detengamos a hacer una serie de consideraciones sobre la no idoneidad para la adopción. 
En primer lugar, hay que señalar que existe un vacío importante en nuestra normativa legal sobre los criterios de idoneidad de los solicitantes de adopción. El Código Civil sólo señala una limitación en cuanto a la edad mínima de los solicitantes, nunca la máxima. Esto genera una discrepancia importante entre los requisitos legales y los técnicos que da pie a que se presenten solicitudes de adopción que, muy probablemente, serán valoradas desfavorablemente, ya que esta restricción legal no puede diluir la obligación de los profesionales de seguir unos criterios técnicos en el proceso de valoración de la idoneidad de las familias para la adopción, basados en el conocimiento de los factores que se han ido evidenciando como precipitadores de situaciones críticas en los procesos adoptivos, aun a pesar de que no se pueda establecer una relación directa entre estos y el fracaso en las adopciones.

Por otra parte, también es necesario señalar una serie de cuestiones relativas a la declaración de no idoneidad y que son importantes para su comprensión (Legaz, E., Crespo, T., 2000). En primer lugar, en la valoración de la idoneidad de las familias para la adopción, hay que considerar su temporalidad, es decir, que ésta se refiere al momento en el que ha sido valorada la familia. Por tanto, no impide que los interesados formulen una nueva solicitud en el futuro, una vez subsanados los problemas que motivaron la desestimación. En relación con esto, la idoneidad hay que entenderla como algo circunstancial: podría ocurrir que una familia que en su día fue declarada idónea no se considere como tal en una revisión posterior, al haber cambiado sus circunstancias personales y/o sociales, o incluso a la inversa. Otra cuestión importante a señalar es el hecho de que una declaración de no idoneidad para la adopción no desacredita ni tiene por qué poner en cuestión el adecuado desenvolvimiento de la familia en otros ámbitos de la vida cotidiana, incluso para el desempeño de la paternidad biológica. Se trata de una valoración específica para unos cometidos muy concretos. Pero, además, la declaración de idoneidad no puede ser entendida genéricamente: puede existir idoneidad para una tipología de menores y no para otra y la no idoneidad podría referirse a la inadecuación entre las expectativas de la familia y sus aptitudes (García Sanz, F., 1999).

Qué duda cabe que, a pesar de estas consideraciones, resulta muy duro para una familia recibir una notificación de este tipo que, si bien no tiene por qué cuestionar la valía de las personas, va a truncar su proyecto de vida, su deseo de ser padres y madres. En este sentido, desde los Servicios de Adopción, y dentro del modelo que se desarrolla actualmente, se procura reflexionar con las familias sobre los aspectos que podrían motivar una declaración de no idoneidad. Así mismo, se les ofrecen distintas alternativas como paso previo a la remisión de los informes desfavorables al Consejo de Adopción, y que incluyen la posibilidad de suspender temporalmente la tramitación del expediente con el fin de subsanar las dificultades detectadas, requerir copia de estos informes y presentar alegaciones al contenido de los mismos, para que sean valorados en las Comisiones Técnicas de la Unidad correspondiente, o bien desistir voluntariamente de la tramitación del expediente si, tras la reflexión efectuada, ellos mismos concluyen que no se dan las condiciones adecuadas para una adopción.

En cualquier caso, si analizamos el número de no idoneidades que se resuelven por parte del Consejo de Adopción de Menores, comprobamos que, durante el año 2006, en la Comunidad Valenciana (datos del Ministerio de Educación, Política Social y Deporte) la cifra se mantuvo alrededor del $6,7 \%$ del total de familias que fueron valoradas, lo cual, teniendo en cuenta lo heterogéneo de las causas posibles de no idoneidad tanto personales como socio-económicas, no parece una cifra elevada, más bien al contrario, sobre todo si se compara con el dato que aportan otros países como Francia que ronda el $20 \%$ y Holanda con un $23 \%$ de no idoneidades.

España es un país relativamente joven en el mundo de la adopción como para poder tener una perspectiva ajustada sobre el éxito o fracaso de los procesos adoptivos. El tiempo 
PORCENTAJE IDONEIDADES 2006

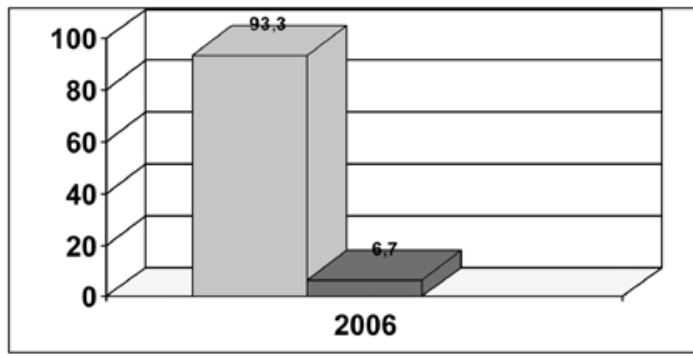

口IDON $\square$ NO IDON

FIGURA 2: Porcentaje de idoneidades y no idoneidades 2006. Fuente: www.mepsyd.es

nos dirá si estas diferencias en el número de no idoneidades son debidas a una excesiva permisividad en la valoración o a que estas familias han llevado a cabo un proceso de preparación que les ha dotado de las herramientas necesarias para hacer frente de forma exitosa a su proyecto adoptivo.

\section{A MODO DE CONCLUSIÓN}

A pesar de los intentos por mejorar los proceso de valoración psico-social, se ha avanzado en la metodología a seguir para lograr unos resultados más fiables, permitiendo una mayor proximidad entre las familias y los profesionales y una mayor profundidad en el abordaje de los distintos temas, pero no se han llegado a identificar patrones o perfiles del buen padre o la buena madre. Incluso las supuestas cualidades y defectos más comúnmente aceptadas por los profesionales deben ser valoradas dentro del contexto en el que se producen (García Sanz, F., 1999).

Por otra parte, nos encontramos con que ha ido variando el perfil de los solicitantes de adopción; cada vez solicitan una adopción más familias con hijos, personas solas, etc. Vemos también que los criterios de idoneidad se han ido modificando y que personas que hubieran sido rechazadas hace 10 ó 15 años actualmente son consideradas idóneas y desarrollan satisfactoriamente su proyecto adoptivo.

Todas estas cuestiones obligan a relativizar de alguna forma los planteamientos técnicos y a no adoptar posturas demasiado rígidas y referidas a un modelo ideal de familia, adoptando las decisiones de forma colegiada, buscando la necesaria objetividad que requiere el poder aunar los deseos de las familias y las necesidades de los menores .

Por otra parte, requiere que se siga investigando sobre aquellas variables que pueden ser factores de riesgo para la adopción o, por el contrario, elementos facilitadores del éxito del proceso adoptivo, sobre las herramientas técnicas para detectarlas $\mathrm{y}$, sobre todo, para poder neutralizar las primeras y potenciar las segundas en el trabajo que se realiza con las familias desde los Servicios de Adopción.

\section{BIBLIOGRAFÍA}

AMORÓS, P. (1987): La adopción y el acogimiento familiar. Narcea.

AMORÓS, P.; FUERTES, J.: La práctica de la adopción. En DE PAUL OCHOTORENA, J., y ARTUA-

BARENA MADARIAGA, M.I. (coords.) (1995): Manual de protección infantil. Barcelona. Masson.

BOWLBY, J. (1985): La separación afectiva. Barcelona. Paidós. 
CYRULNICK, B. (2002): Patitos feos. La resiliencia: una infancia infeliz no determina la vida. Barcelona. Gedisa.

DE PAUL, J.; ARRUABARENA, I. (1995): Manual de protección infantil, cap. 1. 1: La práctica de la adopción. Masson. Barcelona.

GARCÍA SANZ, F. (1999): “El informe psicológico en las adopciones internacionales”. Papeles del psicólogo, $\mathrm{n}^{\mathrm{o}} 73$, pp. 27-35.

GENERALITAT VALENCIANa (1999a): Bases para la valoración psico-social de solicitantes de adopción. Valencia. Conselleria de Bienestar Social.

GENERALITAT VALENCIANA (1999b): Materiales para la preparación de solicitantes de adopción. Valencia. Conselleria de Bienestar Social.

GROZE, V. (1994): Clinical and nonclinical adoptive families of special needs children. Families in society, 75 (2), 90-104.

LEGAZ, E.; CRESPO, T. (2000): "Valoración psicológica de solicitantes de adopción. La propuesta de no idoneidad. Actitudes del valorador y aspectos conceptuales y éticos”. Informació psicològica, $\mathrm{n}^{\circ} 72$, pp. 29-33.

LEGAZ, E. (2003): "Una aproximación a la adopción a través de la teoría del apego". Informació psicològica, 82, pp. 14-20.

MARRONE, M. (2001): La teoría del apego. Madrid. Psimática.

MOYA, C.; ROSSER, A.; GONZÁLEZ, I.: "Formación de familias adoptantes y cambio de actitudes". Actas V Congreso de Infancia Maltratada. Valencia, noviembre 1999.

ROSSER, A.; MOYA, C.; GONZÁLEZ, I. (2000): "Evaluación del impacto de los cursos de formación en las actitudes de las familias adoptantes". VII Congreso de Psicología Social. Oviedo. Noviembre 2000.

TRISEliOTIS, J.; SHIREMAN, J.; HUNDLEBY, M. (1997): Adoption. Theory, policy and practicy. London: Cassell. 